\title{
BMJ Open Quality A pharmacist-led pilot using a performance dashboard to improve psychotropic medication use in a skilled nursing facility
}

\author{
Kristin Bell, ${ }^{1}$ Christine Hartmann, ${ }^{2,3}$ Amy Wisteria Baughman (D) ${ }^{4,5}$
}

To cite: Bell K, Hartmann C, Baughman AW. A pharmacistled pilot using a performance dashboard to improve psychotropic medication use in a skilled nursing facility. BMJ Open Quality 2020;9:e000997. doi:10.1136/ bmjoq-2020-000997

Received 15 April 2020 Revised 1 June 2020 Accepted 21 July 2020

\section{Check for updates}

(c) Author(s) (or their employer(s)) 2020. Re-use permitted under CC BY-NC. No commercial re-use. See rights and permissions. Published by BMJ.

${ }^{1}$ Pharmacy, Veterans Affairs Boston Healthcare System, Boston, Massachusetts, USA ${ }^{2}$ Edith Nourse Rogers Memorial Veterans Hospital, Bedford, Massachusetts, USA

${ }^{3}$ Public Health, University of Massachusetts Lowell, Lowell, Massachusetts, USA

${ }^{4}$ Geriatrics and Extended Care, Veterans Affairs Boston Healthcare System, Boston, MA, United States

${ }^{5}$ Harvard Medical School, Boston, MA, United States

Correspondence to Dr Amy Wisteria Baughman; awbaughman@mgh.harvard. edu

\section{ABSTRACT}

Objectives Inappropriate use of psychotropic medications in the elderly, particularly those with dementia, is a critical safety and quality concern. This pilot quality improvement study used a novel Department of Veterans Affairs (VA) Psychotropic Drug Safety Initiative performance dashboard (PDSI dashboard) to implement a pharmacistled intervention to improve psychotropic medication prescribing practices in a VA skilled nursing facility (SNF). While clinical dashboard data have become commonplace, literature describing successful implementation for improved clinical care is scant.

Methods This study took place from November 2015 to February 2016 at a 112-bed VA SNF. A pharmacist used the PDSI dashboard to identify 'actionable' patients with potentially inappropriate psychotropic prescribing and then completed chart reviews to confirm clinical indications. The pharmacist provided recommendations to providers for dose reductions or deprescribing via in-person communication and notes written in the electronic medical record. SNF providers completed anonymous surveys about their experience in receiving recommendations. Results Over a 5-month period, the PDSI dashboard identified 21 patients with potentially inappropriate psychotropic medication use, with approximately one new patient identified each week. Prescribing recommendations were accepted $66 \%$ of the time. All seven SNF providers reported that recommendations were helpful in improving their psychotropic prescribing practices.

Conclusions The PDSI dashboard was efficient and effective in identifying patients at risk for inappropriate use of psychotropic medications. A clinical pharmacist was essential for implementing and communicating recommendations from the dashboard to providers.

\section{INTRODUCTION}

Inappropriate use of psychotropic medications in the elderly, particularly those living with dementia, is a critical safety and quality concern across the nation. Psychotropic misuse can expose patients to serious medical side effects including death and worsened cognitive functioning. ${ }^{1-4}$ Despite this potential harm, several studies have shown widespread use of psychotropic medications among patients living in skilled nursing facilities $(\mathrm{SNFs}){ }^{4-8}$ The high prevalence of psychotropic use raises concern for inappropriate prescribing. For example, within the Veterans Affairs (VA) specifically, one study found that over half of veterans on psychotropic medications in the outpatient setting did not have a concurrent psychiatric diagnosis.

Psychotropic medication use in patients with dementia raises particular concerns. These patients are frequently treated with psychotropic medications for neuropsychiatric symptoms. ${ }^{10}$ However, certain psychotropics, such as antipsychotic medications, are associated with increased mortality and have limited efficacy for behavioural symptoms of dementia. ${ }^{1-13}$ Additionally, these medications have significant side effects such as drowsiness, increased fall risk, cerebrovascular accidents and greater risk of cognitive decline, which can exacerbate symptoms of dementia. 31415

Nevertheless, antipsychotic use for behavioural symptoms of dementia continues. A 2012 study of older adults in VA nursing homes found that $26 \%$ of patients were prescribed antipsychotics during a 90-day or more inpatient stay. Only $59 \%$ of these patients had an evidence-based indication for antipsychotic use. ${ }^{16}$ Furthermore, while many studies have found interventions to decrease psychotropic prescribing, few have shown reductions in clinical outcomes such as mortality, falls and adverse events. ${ }^{17}$

Deprescribing is an important strategy that may help address psychotropic misuse. Deprescribing refers to the process of safely and effectively stopping a medication that has minimal benefit. ${ }^{18}$ While deprescribing is an important strategy for improving prescribing practices, the literature on successful deprescribing interventions is now just emerging. Lack of time and expertise of potential harms of medications are specific barriers 
to deprescribing for providers. ${ }^{19}$ These barriers may be addressed by a pharmacist-led intervention. Studies have shown that collaboration between clinicians and pharmacists can help optimise medication regimens and appropriately deprescribe medications for older adult patients. ${ }^{19-21}$

Another potential tool to facilitate deprescribing may be the VA Psychotropic Drug Safety Initiative (PDSI) ${ }^{22}$ which aims to improve the safety and effectiveness of medication use for veterans with mental health problems. The PDSI is a nationwide VA quality improvement (QI) performance dashboard that identifies concerning patterns of psychotropic medication prescribing. However, many questions remain around effective clinical dashboard use and implementation. Integrating the PDSI dashboard into clinical practice requires training on the measures and website use, as well as changes in workflow for log-in and dashboard review. Finally, successful PDSI dashboard implementation requires identifying the appropriate end users. To address these questions, we designed a QI study involving a clinical pharmacist to implement the PDSI dashboard.

Identifying strategies to improve prescribing practices in SNFs requires continued innovation and exploration of multimodal strategies such as the PDSI dashboard. This study evaluated a pharmacist-led intervention that used the PDSI dashboard to improve prescribing practices of psychotropic medications in a VA SNF.

\section{METHODS}

\section{Study design and setting}

The VA is the largest integrated healthcare system in the USA with over nine million enrolled Veterans. This QI study took place at a VA Community Living Center (CLC), a 112-bed VA SNF in New England. The SNF included a 65-bed transitional care unit, a 15-bed hospice unit and a 32-bed long-term care unit. Clinical providers included four physicians, two physician assistants and one nurse practitioner. The study took place over 22 weeks; the baseline period was from 20 November 2015 to 5 February 2016 and the intervention period was from 6 February
2016 to 22 April 2016. The VA Boston Healthcare System Institutional Review Board approved this study.

\section{Study population and data source}

All patients in our SNF were screened by the PDSI dashboard. The dashboard identified patients who flagged for additional pharmacologic review based on the dashboard psychotropic measures. These patients were prescribed psychotropic medications and either had a diagnosis of dementia on their problem list or were 75 years or older.

\section{Description of the intervention}

The PDSI dashboard is a nationwide VA QI tool that was launched in September 2013 and provides real-time monitoring of prescribing practices by identifying patients who may benefit from additional medical review based on pharmacy administration data. In October 2015, the PDSI added seven measures specific for older adult patients in VA SNFs around anticholinergic, antihistamine, antipsychotic and antipsychotic medication use (table 1).

The PDSI dashboard displays data via a web-based dashboard interface and is available to all VA facilities and VA providers. Overall facility trends can be accessed by any VA employee, however, specific patient-level data requires approval that can be easily requested on the dashboard website. For each measure, the dashboard shows quarterly prescribing trends, flags 'actionable' patients, and provides evidence-based prescribing recommendations. The PDSI dashboard aims to facilitate optimal prescribing and deprescribing by proactively identifying patients on a daily basis and encouraging re-evaluation of the risks and benefits of long-term use of psychotropic medications.

The PDSI dashboard was selected as an intervention due to the recent release of new measures specific for VA SNFs as well as limited research describing successful implementation of clinical dashboards. This work was designed and completed by a geriatric pharmacy resident as part of their research curriculum. The pharmacy effort for all aspects of the study involved 0.1 full-time equivalent (FTE). All SNF clinicians were notified of the study intervention and were provided a brief overview of

Table 1 PDSI phase 2 measures for CLCs

PDSI CLC measure Definition

Anticholinergic antidepressant

Anticholinergic polypharmacy

Antihistamine use

Antipsychotic use with dementia

Antipsychotic use monitoring

Benzodiazepine use with dementia

Benzodiazepine or sedative use
Anticholinergic antidepressant use in patients who are 75 or older

Two or more anticholinergic medications in patients who are 75 or older Antihistamine use in patients who are 75 or older

Antipsychotic use in patients with dementia, without diagnosis of psychosis, schizophrenia or bipolar disorder

Lack of glucose or A1c in patients who are 75 or older taking antipsychotic medications

Benzodiazepine use in patients with dementia who are 75 or older Benzodiazepine use in patients who are 75 or older

CLC, Community Living Center; PDSI, Psychotropic Drug Safety Initiative. 
the PDSI dashboard and the seven PDSI psychotropic measures during a monthly staff meeting.

During both the baseline and the intervention period, a pharmacist reviewed patients identified by the PDSI dashboard and evaluated prescribing indications and medical monitoring for each psychotropic medication. During the intervention period only, the pharmacist made recommendations for potential dose reduction or deprescribing based on the evidence-based prescribing guidelines provided by the dashboard. The pharmacist communicated the recommendations to the patient's provider via a cosigned note in the electronic medical record and a face-to-face conversation within 5 days of entering the electronic note.

\section{Outcome measures and clinician survey}

The first outcome measure was the number of new actionable patients identified by the dashboard before and after the intervention. Patients who had previously been identified by the dashboard but continued to flag as actionable were not considered newly actionable. Our hypothesis was that the intervention would decrease new actionable patients as providers improved their prescribing practices.

Four weeks after the pharmacist provided the recommendations, research staff reviewed the medical record to evaluate the recommendation uptake. We also surveyed participating clinicians with a brief, anonymous online questionnaire one week after the intervention period ended. Questions asked about the effectiveness of the intervention and what the preferred communication mode was for receiving recommendations.

\section{Patient and public involvement}

Patients or the public were not involved in the design, or conduct, or reporting or dissemination plans of our research.

\section{RESULTS}

The PDSI dashbard identified 21 patients as actionable, with an average of one new patient each week. All patients were male and the average age was 83 years (range, 69-95 years). Over half of these patients were admitted for long-term care $(59 \%)$. Five patients were admitted for transitional care $(27 \%)$, and three patients were admitted to the hospice unit. Six out of seven PDSI measures triggered several times during the study period for a total of 23 triggers (table 2). One PDSI measure (antipsychotic medication monitoring) had zero triggers.

There were 15 new actionable patients identified in the baseline period, and 6 new actionable patients identified in the intervention period. This decrease was not significant, likely due to small sample size $(p=0.09$ with a one-tailed t-test assuming unequal variance). Six patients identified during the baseline period continued to remain actionable during the intervention period and so the PDSI dashboard identified a total of 12 actionable patients during the intervention period.

Of these 12 patients in the intervention period, recommendations could be made for psychotropic medication adjustment in nine of these patients. Providers implemented six recommendations and declined three, resulting in $66 \%$ recommendation uptake. Reasons for declining included behavioural issues during prior attempts to taper, family preference for current treatment regimen and high symptom burden (eg, hydroxyzine for pruritus). The remaining three actionable patients did not receive recommendations because a change in prescribing practice was not necessary. For example, two of these patients were undergoing radiation treatment and were prescribed one-time use benzodiazepines for anxiety only during treatment.

All seven providers responded to the survey. Providers had a mixed preference in favoured communication mode for recommendations. Two providers preferred face-to-face communication, two preferred electronic note communication and three preferred both. All providers preferred a pharmacist notification for actionable patients rather than having to independently $\log$ onto the PDSI dashboard on their own. Additionally, all providers found the recommendations to be helpful in improving their psychotropic prescribing practices. Five out of seven clinicians reported learning information that would change their future prescribing behaviour. Six out of seven clinicians reported that they would like the PDSI recommendations to continue in the future (one clinician gave a neutral response).

Table 2 PDSI dashboard measures and psychotropic medications identified during the study period

\begin{tabular}{lcl}
\hline PDSI measure & Number of measures that triggered & Psychotropic medications \\
\hline Anticholinergic antidepressant & 4 & Paroxetine, Noritriptyline \\
\hline Anticholinergic polypharmacy & 5 & Oxybutynin, Tropsium, Atropine, Meclizine \\
Antihistamine use & 6 & Hydroxyzine, Diphenhydramine, Loratidine \\
Antipsychotic use with dementia & 3 & Risperidone, Olanzapine, Quetiapine, Haloperidol \\
Antipsychotic use monitoring & 0 & \\
Benzodiazepine use with dementia & 3 & Clonazepan, Diazepan, Lorazepan, Temazepan \\
Benzodiazepine or sedative use & 2 & Zolpidem, Trazodone \\
\hline
\end{tabular}

PDSI, Psychotropic Drug Safety Initiative. 


\section{DISCUSSION}

This study provides preliminary evidence for the effectiveness of a pharmacist-led intervention using a clinical performance dashboard as an effective tool for identifying and communicating about SNF patients receiving potentially inappropriate psychotropic medications. A collaborative approach with a pharmacist providing recommendations both face-to-face as well as by electronic medical record resulted in improved prescribing practices as reflected by a high uptake of recommendations $(66 \%)$. This approach was also independently well received by clinicians in a follow-up survey, with most clinicians reporting learning information that would change their future prescribing.

Several studies have shown that inappropriate psychotropic medication prescribing continues to be a common and significant issue in the elderly population due to adverse effects, functional impairment and geriatric syndromes. ${ }^{513} 15$ However, fewer studies have identified potential solutions to address these issues, especially for patients in SNFs. A 2016 Cochrane review of 12 studies evaluating interventions to improve prescribing for patients in care homes (SNFs or assisted-living facilities) found weak evidence that some interventions could identify medication-related problems and improvements in medication appropriateness, but no evidence to support interventions that reduced patient-related outcomes such as adverse drug events or death. ${ }^{17}$ Furthermore, robust conclusions could not be drawn due to heterogeneity of the studies.

Similar to our results, appropriate prescribing was improved in several studies. ${ }^{23-27}$ García-Gollarte et al found that an educational intervention for SNF physicians involving didactics and workshops improved appropriate prescribing using the Screening Tool of Older Persons Prescriptions (STOPP) and Screening Tool to Alert Doctors to Right Treatment (START) criteria. ${ }^{26}$ The total time dedicated to the education intervention and the delivery mechanism (eg, lectures or educational materials) was not defined, limiting comparison to our work.

Frankenthal et al evaluated an intervention in a longterm care facility where a pharmacist reviewed prescriptions using the STOPP and START criteria at 6 and 12 months. However, recommendations were only provided to a chief physician and not all physicians, limiting the long-term educational impact. ${ }^{25}$

A 2004 study by Crotty et al using a transition pharmacist coordinator who facilitated evidence-based review of medications at hospital discharge and on admission to the long-term care facility found improvements in the medication appropriateness index. ${ }^{24}$ This intervention, however, was resource-intensive and involved medication reconciliation of medications at discharge, another review at 10-14 days, and then a follow-up conference with the intervention pharmacist, long-term care pharmacist, nurse, and physician. Another study involving a pharmacist intervention using a telephone-call intervention, where a geriatric pharmacist reviewed utilisation data and contacted prescribers, found improvements in prescribing. ${ }^{28}$ This study, however, as compared with ours did not provide clinicians with written recommendations which may have limited the educational and long-term impact of the intervention.

Studies have shown several barriers to successful deprescribing, including limited time and knowledge. ${ }^{19}$ Many clinicians find the process of deprescribing difficult since it requires evaluating whether treatment may cause more harm than benefit. ${ }^{29}$ In addition, patients often feel pressured by family or health professionals to start or continue a medication. ${ }^{31}$ Many also worry about symptom return if they were taper or stop their medications. ${ }^{10}$ This intervention addressed many of these barriers by prompting evaluation of specific medications and providing clear evidence-based recommendations. Importantly, this intervention also allowed for decisions to be highly patient centred.

Our intervention also involved the use of a clinical dashboard. While clinical performance dashboards have become increasingly common with the advent of electronic healthcare records, it is still unclear how best to use these tools to improve care. Not all providers have found dashboards helpful even when the information may be clinically relevant. A small National Health System study surveyed 21 mental health professionals about a clinical dashboard for assessments and falls in acute older adult care; researchers found that only $38 \%$ of respondents found the dashboard helpful and only $48 \%$ perceived the metrics to be useful. ${ }^{32}$ In contrast to this, our study found that all seven providers appreciated the dashboard information both from a clinical and educational standpoint. However, we believe that the pharmacist intervention was essential for enabling the use of the PDSI dashboard because of the time involved with dashboard login and access.

For optimal implementation, QI interventions should not increase clinician workload, as adoption may otherwise be limited. A 2014 systematic review of 11 dashboard studies found that easily accessible dashboards (eg, screensaver) were associated with improved care processes and patient outcomes. ${ }^{33}$ The PDSI dashboard is not easily accessible and required two steps for access, including a one-time only administrative approval step where the VA provider must be approved to access deidentified data. Additionally, each time the database is accessed, a login with password is required. These additional steps were facilitated by the pharmacist, which was likely necessary for success.

On average the dashboard only identified one new patient per week, which resulted in approximately 60 minutes of chart review and documentation. Our 112-bed facility is of average size for a SNF. ${ }^{34}$ Given the potential harm of inappropriate psychotropic use and the increasingly important role of psychotropic prescribing as a nationally tracked quality measure in SNF care, ${ }^{35}$ we believe that this weekly 1 hour commitment of pharmacist 
time is highly worthwhile. In addition, our study pharmacist found this work to be interesting and important for patient care; it also provided a meaningful opportunity to collaborate with the care team.

This study's small sample size, short timeframe and single site involvement may limit this study's generalisability. However, this dashboard tool is freely available to all VA CLCs, and no formal training is necessary for dashboard use. This report describes a strategy for implementation that can be easily replicated.

Moving forward, our work supports the importance of the pharmacist-provider collaboration for deprescribing as it addresses several key barriers and also enables a highly patient-centred approach. This work also highlights the utility of a clinical dashboard for tracking quality measures such as psychotropic use and identifying specific areas for improvement.

Future research should evaluate how psychotropic deprescribing and dose reduction impacts patientfocused clinical measures such as physical and cognitive functioning and morbidity. Other important areas of evaluation should include challenges that providers may face during the process of deprescribing. For example, SNF providers may feel pressured by nursing staff to prescribe antipsychotics for behavioural problems, particularly in patients with dementia. ${ }^{30}$ Finally, as reflected by the systemic reviews, future work should include rigorously designed high-quality studies including randomised controlled trials to determine which interventions can result clearly defined improved patient outcomes.

\section{CONCLUSION}

This pilot QI study provides important feasibility information about using an innovative clinical performance dashboard in conjunction with a pharmacist-led deprescribing intervention. It demonstrates that a performance dashboard can be used to improve prescribing for psychotropic medications-an important area of misuse in the SNF population. Similar to other studies, this study confirmed that a pharmacist-physician relationship is crucial in accomplishing medication deprescribing. This collaborative model facilitates challenging decisionmaking that ultimately can improve patient care.

Twitter Amy Wisteria Baughman @amywisteria

Contributors KB contributed to the conception, acquisition, analysis and interpretation of data for this study. She also participated in the drafting of the manuscript. AWB contributed to the conception, acquisition, analysis and interpretation of data. She was also involved in the writing and revision of the manuscript. $\mathrm{CH}$ was involved in the writing and revision of the manuscript.

Funding The authors have not declared a specific grant for this research from any funding agency in the public, commercial or not-for-profit sectors.

Competing interests None declared.

Patient consent for publication Not required.

Provenance and peer review Not commissioned; externally peer reviewed.

Data availability statement Data are available upon reasonable request.

Open access This is an open access article distributed in accordance with the Creative Commons Attribution Non Commercial (CC BY-NC 4.0) license, which permits others to distribute, remix, adapt, build upon this work non-commercially, and license their derivative works on different terms, provided the original work is properly cited, appropriate credit is given, any changes made indicated, and the use is non-commercial. See: http://creativecommons.org/licenses/by-nc/4.0/.

\section{ORCID iD}

Amy Wisteria Baughman http://orcid.org/0000-0002-1275-3769

\section{REFERENCES}

1 Fick DM, Cooper JW, Wade WE, et al. Updating the beers criteria for potentially inappropriate medication use in older adults: results of a US consensus panel of experts. Arch Intern Med 2003;163:2716-24.

2 Gurwitz JH, Field TS, Judge J, et al. The incidence of adverse drug events in two large academic long-term care facilities. Am J Med 2005;118:251-8.

3 Rosenberg PB, Mielke MM, Han D, et al. The association of psychotropic medication use with the cognitive, functional, and neuropsychiatric trajectory of Alzheimer's disease. Int J Geriatr Psychiatry 2012;27:1248-57.

4 Liperoti R, Sganga F, Landi F, et al. Antipsychotic drug interactions and mortality among nursing home residents with cognitive impairment. J Clin Psychiatry 2017;78:e76-82.

5 Bronskill SE, Anderson GM, Sykora K, et al. Neuroleptic drug therapy in older adults newly admitted to nursing homes: incidence, dose, and specialist contact. J Am Geriatr Soc 2004;52:749-55.

6 Helvik A-S, Šaltyte Benth J, Wu B, et al. Persistent use of psychotropic drugs in nursing home residents in Norway. BMC Geriatr 2017;17:52.

7 Huber M, Kölzsch M, Rapp MA, et al. Antipsychotic drugs predominate in pharmacotherapy of nursing home residents with dementia. Pharmacopsychiatry 2012;45:182-8.

8 Resnick B, Kolanowski A, Van Haitsma K, et al. Current psychotropic medication use and contributing factors among nursing home residents with cognitive impairment. Clin Nurs Res 2019;1054773819 838678:105477381983867.

9 Wiechers IR, Kirwin PD, Rosenheck RA. Increased risk among older veterans of prescribing psychotropic medication in the absence of psychiatric diagnoses. Am J Geriatr Psychiatry 2014;22:531-9.

10 Wouters H, Quik EH, Boersma F, et al. Discontinuing inappropriate medication in nursing home residents (DIM-NHR study): protocol of a cluster randomised controlled trial. BMJ Open 2014;4:e006082.

11 Kuehn BM. Fda warns antipsychotic drugs may be risky for elderly. JAMA 2005;293:2462.

12 Gill SS, Bronskill SE, Normand S-LT, et al. Antipsychotic drug use and mortality in older adults with dementia. Ann Intern Med 2007;146:775-86.

13 Maust DT, Kim HM, Seyfried LS, et al. Antipsychotics, other psychotropics, and the risk of death in patients with dementia: number needed to harm. JAMA Psychiatry 2015;72:438-45.

14 Hsu W-T, Esmaily-Fard A, Lai C-C, et al. Antipsychotics and the Risk of Cerebrovascular Accident: A Systematic Review and Meta-Analysis of Observational Studies. J Am Med Dir Assoc 2017;18:692-9.

15 Johnell K, Jonasdottir Bergman G, Fastbom J, et al. Psychotropic drugs and the risk of fall injuries, hospitalisations and mortality among older adults. Int J Geriatr Psychiatry 2017;32:414-20.

16 Gellad WF, Aspinall SL, Handler SM, et al. Use of antipsychotics among older residents in Va nursing homes. Med Care 2012;50:954-60.

17 Alldred DP, Kennedy M-C, Hughes C, et al. Interventions to optimise prescribing for older people in care homes. Cochrane Database Syst Rev 2016;2:CD009095.

18 Reeve E, Shakib S, Hendrix I, et al. Review of deprescribing processes and development of an evidence-based, patient-centred deprescribing process. Br J Clin Pharmacol 2014;78:738-47.

19 Anderson K, Stowasser D, Freeman C, et al. Prescriber barriers and enablers to minimising potentially inappropriate medications in adults: a systematic review and thematic synthesis. BMJ Open 2014;4:e006544.

20 Frank C. Deprescribing: a new word to guide medication review. CMAJ 2014;186:407-8.

21 Hilmer SN, Gnjidic D, Le Couteur DG. Thinking through the medication list - appropriate prescribing and deprescribing in robust and frail older patients. Aust Fam Physician 2012;41:924-8.

22 Wiechers IR. Improving psychopharmacological care for older veterans: implementation of phase 2 of the psychotropic drug safety initiative. Am J Geriatr Psychiatry 2016;24:S155-6. 
23 Crotty M, Halbert J, Rowett D, et al. An outreach geriatric medication Advisory service in residential aged care: a randomised controlled trial of case conferencing. Age Ageing 2004;33:612-7.

24 Crotty M, Rowett D, Spurling L, et al. Does the addition of a pharmacist transition coordinator improve evidence-based medication management and health outcomes in older adults moving from the hospital to a long-term care facility? results of a randomized, controlled trial. Am J Geriatr Pharmacother 2004;2:257-64.

25 Frankenthal D, Lerman Y, Kalendaryev E, et al. Intervention with the screening tool of older persons potentially inappropriate prescriptions/screening tool to alert doctors to right treatment criteria in elderly residents of a chronic geriatric facility: a randomized clinical trial. J Am Geriatr Soc 2014;62:1658-65.

26 García-Gollarte F, Baleriola-Júlvez J, Ferrero-López I, et al. An educational intervention on drug use in nursing homes improves health outcomes resource utilization and reduces inappropriate drug prescription. J Am Med Dir Assoc 2014;15:885-91.

27 Pitkälä KH, Juola A-L, Kautiainen $\mathrm{H}$, et al. Education to reduce potentially harmful medication use among residents of assisted living facilities: a randomized controlled trial. J Am Med Dir Assoc 2014;15:892-8.

28 Monane M, Matthias DM, Nagle BA, et al. Improving prescribing patterns for the elderly through an online drug utilization review intervention: a system linking the physician, pharmacist, and computer. JAMA 1998;280:1249-52.

29 Farrell B, Tsang C, Raman-Wilms L, et al. What are priorities for deprescribing for elderly patients? capturing the voice of practitioners: a modified Delphi process. PLoS One 2015;10:e0122246.

30 Garfinkel D, Mangin D. Feasibility study of a systematic approach for discontinuation of multiple medications in older adults: addressing polypharmacy. Arch Intern Med 2010;170:1648-54.

31 Reeve E, To J, Hendrix I, et al. Patient barriers to and enablers of deprescribing: a systematic review. Drugs Aging 2013;30:793-807.

32 Daley K, Richardson J, James I, et al. Clinical dashboard: use in older adult mental health wards. Psychiatrist 2013;37:85-8.

33 Dowding D, Randell R, Gardner P, et al. Dashboards for improving patient care: review of the literature. Int J Med Inform 2015;84:87-100.

34 Centers for Medicare and Medicaid Services. Nursing home data compendium 2015 edition, 2015. https://www.cms.gov/Medicare/ Provider-Enrollment-and-Certification/CertificationandComplianc/ Downloads/nursinghomedatacompendium_508-2015.pdf

35 U.S. Centers for Medicare and Medicaid services quality measures, 2019. Available: https://www.cms.gov/Medicare/Quality-InitiativesPatient-Assessment-Instruments/NursingHomeQualitylnits/ $\mathrm{NHQIQualityMeasures.html}$ 